\section{apresentação de caso}

Táki A. Cordás

Arnaldo P. Lopes Filbo

Adriano Segal

\author{
Ambulatório de Obesidade e \\ Co-morbidez Psiquiátrica \\ (AMBESO) do Ambulatório de \\ Bulimia e Transtornos \\ Alimentares (AMBULIM), \\ Instituto de Psiquiatria do \\ Hospital das Clinicas da \\ Faculdade de Medicina da \\ Universidade de São Paulo, SP.
}

Recebido em 20/08/02

Revisado em 30/03/04 e 14/06/04

Aceito em 23/06/04

\section{Transtorno Alimentar e Cirurgia Bariátrica: Relato de Caso}

\begin{abstract}
RESUMO
Objetivo: Os autores discutem, a partir de um relato de caso e de uma revisão da literatura, as implicaçōes de uma operação bariátrica sobre o comportamento alimentar. Método: É apresentado o caso clínico de uma paciente submetida a uma operação bariátrica e que apresentou alterações comportamentais alimentares semelhantes às de uma anorexia nervosa, mas não apresentando peso abaixo do normal, o que levou a um diagnóstico de transtorno alimentar não especificado. Este caso é analisado em comparação com situaçōes semelhantes na literatura. Discussão: Os dados da literatura são discutidos de acordo com aqueles apresentados no caso relatado e procura-se, a partir daí, obter uma conduta prudente frente aos candidatos à cirurgia bariátrica e no seguimento pós-operatório, analisando os possíveis riscos envolvidos. (Arq Bras Endocrinol Metab 2004;48/4:564-571)
\end{abstract}

Descritores: Comportamento alimentar; Transtorno alimentar; Cirurgia bariátrica; Anorexia nervosa

\section{ABSTRACT}

Eating Disorder and Bariatric Surgery: A Case Report.

Objective: The authors discuss, based on a case report and on a literature review, the implications of a bariatric surgery for obesity on the eating behavior. Method: A case report is presented in which a patient was submitted to a bariatric surgery and show altered eating behavior like in anorexia nervosa, but without low weight, leading to the diagnostic of an otherwise unspecified eating disorder. This case is compared with the literature review. Discussion: Literature information is discussed according to data from the present case and based on this, aiming to establish a prudent conduct with bariatric surgery candidates and their follow up, analyzing the possible risks involved. (Arq Bras Endocrinol Metab 2004;48/4:564-571)

Keywords: Eating behavior; Eating disorder; Bariatric surgery; Anorexia nervosa

PARENTEMENTE, NADA PODE SER tão distante, ou sugerir tanto uma
e pacientesão clínica, quanto pacientes com Anorexia Nervosa de um lado
cente uso das cirurgias bariátricas no tratamento da obesidade, relatos de
evolução pós-cirúrgica para Anorexia Nervosa e Bulimia Nervosa começam
a ser descritos.
Cirurgia bariátrica é a medida mais efetiva no tratamento da obesi-
dade mórbida. Uma adequada avaliação psiquiátrica pré-cirúrgica é essen-
cial para o diagnóstico e adequado tratamento precoce de um transtorno
alimentar prévio. Powers e cols. (10), num grupo de 116 pacientes sub-
metidos à cirurgia gástrica restritiva, estudados por 10 anos, encontraram
episódios bulímicos em $52 \%$ dos casos, Transtorno da Compulsão Alimen- 
tar Periódica (Binge Eating Disorder) em 16\% e Síndrome do Comer Noturno em 10\%.

Hsu e cols. (12), entrevistando pacientes do sexo feminino submetidas à gastroplastia vertical, evidenciaram que alterações do comportamento alimentar précirúrgico, como Bulimia Nervosa, Síndrome do Comer Noturno e ingestão compulsiva de grandes quantidades de líquidos, estavam presentes em $75 \%$ dos casos e que a cirurgia obteve sucesso na redução do peso, mas não foi suficiente para remitir o comportamento alimentar prévio. Uma melhora do comportamento alimentar pode ocorrer precocemente, após a operação, mas o retorno dos transtornos alimentares em pacientes submetidos à cirurgia de bypass gástrico num seguimento de até 3 anos levam a um reganho de peso rápido, geralmente 2 anos após o procedimento cirúrgico (13). Kalarchian e cols. (11) descrevem que cerca de $40 \%$ de 64 obesos mórbidos candidatos à cirurgia de bypass gástrico apresentavam episódios bulímicos.

Maddi e cols. (24) observaram um decréscimo na psicopatologia presente em pacientes operados, utilizando o MMPI-2 (Minnesota Multiphasic Personality Inventory, Second Edition). Entretanto, o MMPI-2 contempla os transtornos psiquiátricos de uma maneira geral, mas não os transtornos alimentares.

Bonne e cols. (1) referem dois casos de Anorexia Nervosa em homens jovens (19 e 27 anos) após gastroplastia vertical para o tratamento de obesidade mórbida. Ambos com dificuldades prévias de relacionamento interpessoal e o mais jovem homossexual, achado freqüentemente relatado entre homens com transtornos alimentares (2-4).

Atchinson e cols. (5) descrevem duas mulheres em Adelaide, Austrália, que desenvolveram Anorexia Nervosa no seguimento de um ano após cirurgia de redução gástrica com grampeamento (stapler), e sugerem que aspectos psicológicos, como dependência e o uso de alimentos como "conforto emocional", podem predispor ao desenvolvimento de Anorexia Nervosa.

Scioscia e cols. (6) descrevem o caso de uma mulher que desenvolveu Anorexia Nervosa subtipo purgativo, abuso de drogas (maconha, álcool e LSD) e depressão no seguimento de dois anos após a cirurgia de bypass gástrico. Uma revisão de seu prontuário précirúrgico não evidenciava história positiva para qualquer quadro psiquiátrico, provavelmente em função de uma anamnese sumária, onde não foram indagados aspectos importantes de seus antecedentes pessoais. Os autores propõem uma história psiquiátrica pormenorizada e a aplicação de escalas para transtornos alimentares como o Eating Disorders Inventory (EDI) e o Eating Disor ders Examination (EDE) na avaliação pré-operatória, a fim de se ter diagnosticados possíveis transtornos alimentares prévios à operação e observar mais atentamente tais pacientes no seguimento pós-cirúrgico.

Algazi (7) propõe a formação de grupos de suporte após a operação incluindo pacientes, pais e cônjuges, com reuniões periódicas utilizando técnicas de aconselhamento e resolução de problemas. Estes grupos visam uma melhor aderência ao tratamento, com orientações quanto à nutrição pós-cirúrgica, exercícios físicos, dietas e detecção precoce de quadros psiquiátricos. Waters e cols. (8) sugerem a formação de grupos pré e pós-cirúrgicos com finalidades semelhantes.

Em outras palavras, não é possível prever todos os casos onde possa ocorrer transtornos alimentares ou outros transtornos psiquiátricos no pósoperatório, mas a existência de tais patologias no préoperatório deve nos alertar para maior observação do paciente após a operação.

Além disso, os transtornos alimentares prévios tendem a sofrer uma parada transitória ou modificação em sua manifestação após a operação, como, por exemplo, o paciente substituir os binge eatings por grazing (comer pequenas quantidades de alimentos em longo tempo) (25).

O padrão alimentar desordenado tende a persistir após a operação e pode agravar a condição clínica do paciente, além de, no longo prazo, facilitar o reganho de peso. As técnicas psicoterapêuticas cognitivo-comportamentais podem ser muito úteis neste caso, tratando diretamente o comportamento alimentar e evitando complicações.

A seguir, relatamos um caso clínico que cursou com um transtorno alimentar no seguimento pósoperatório.

\section{RELATO DO CASO}

MDA, 40 anos, sexo feminino, branca, do lar, solteira, natural e procedente de São Paulo, SP.

\section{Queixa e Duração}

"Eu quero emagrecer mais, e tenho só mais 1 mês para fazer isso".

\section{História da Moléstia Atual}

Paciente procurou tratamento psiquiátrico particular em fevereiro de 1999, 11 meses após operação bariátrica, técnica de Capella (bypass gástrico), encaminhada pela psicóloga da equipe que realizou a operação. Neste período perdeu $70 \mathrm{~kg}$, representando esta variação mais de $50 \%$ de seu peso inicial. 
Durante quatro meses de tratamento psiquiátrico, sofreu uma internação de 3 semanas em clínica privada devido a quadro de vômitos auto-induzidos após a alimentação, recusa alimentar, distorção da imagem corporal (continuava se achando obesa apesar de estar com um peso adequado), medo intenso de ganho de peso, episódios documentados de hipoglicemia que culminavam com quedas e desmaios freqüentes.

Foi medicada com doses progressivas de fluoxetina até $80 \mathrm{mg} /$ dia, este um antidepressivo, associada à periciazina $20 \mathrm{mg} /$ dia, um neuroléptico com importante ação sobre a impulsividade, após serem formuladas as hipóteses diagnósticas descritas adiante. Não se aventou a possibilidade de estar ocorrendo alguma disfunção gástrica decorrente da própria operação, pois os outros sintomas e comportamentos descartavam esta hipótese.

A paciente apresentava, neste período, irregularidade menstrual e peso variando entre discreto sobrepeso e dentro dos limites da normalidade.

Foi encaminhada ao AMBESO/AMBULIM por falha terapêutica.

Devido ao histórico prévio, decidiu-se pela internação da paciente já na primeira consulta, realizada em 18/06/1999.

\section{Antecedentes Pessoais}

Paciente com história de obesidade desde a infância, com diferentes tratamentos em clínicas de emagrecimento e spas, além de dietas comerciais e uso de várias medicações anti-obesidade, prescritas ou não por médico.

Chegou ao quadro de obesidade grau III (Índice de Massa Corpórea (IMC) $>39,9 \mathrm{~kg} / \mathrm{m}^{2}$ ) no início da idade adulta e seu IMC máximo chegou a $56,5 \mathrm{~kg} / \mathrm{m}^{2}$, quando da operação bariátrica. Neste período, previamente à operação, relata quadro compatível com Bulimia Nervosa, segundo o DSM-IV (20) (tabela 1).

Refere-se uma pessoa impulsiva, com brigas freqüentes com familiares e amigos e dificuldades de relacionamento interpessoal. Nega relacionamento afetivo estável. A paciente afirma que costumeiramente agredia fisicamente amigos devido a forte prazer que esta atitude proporcionava, e que estas agressões ocorriam com a concordância dos mesmos.

Com seus irmãos, costumava torturar pequenos animais domésticos até a eventual morte de alguns deles.

A paciente relata ainda fantasias sádicas e interesse em sites de sado-masoquismo, que costumava freqüentar pela Internet.

Além da medicação anteriormente descrita, a paciente deveria fazer uso de suplementação vitamínica e de ferro, não o fazendo devido ao medo de engordar.

\section{Antecedentes Familiares}

Familiares com sobrepeso. Nega história de transtornos psiquiátricos na família, embora descreva,

Tabela 1. Critérios diagnósticos para Bulimia Nervosa (DSM-IV).

\section{Bulimia Nervosa:}

Episódios bulímicos recorrentes. Um episódio bulímico é caracterizado por ambos os critérios abaixo:

- comer, num discreto período de tempo (por exemplo, dentro de um período de duas horas), uma quantidade de alimento que é definitivamente maior que a maioria das pessoas comeriam durante um período similar de tempo e sob circunstâncias similares

- uma sensação de falta de controle sobre o comer durante o episódio (por exemplo, um sentimento de que não pode parar de comer ou controlar o que e o quanto é ingerido)

Comportamento compensatório recorrente inapropriado para prevenir o ganho de peso, tal como induzir vômitos, uso de laxantes, diuréticos, enemas ou outros medicamentos, jejum ou exercício físico excessivo

Os episódios bulímicos e os comportamentos compensatórios inapropriados ocorrem ambos, em média, pelo menos duas vezes por semana por três meses.

A auto-avaliação é excessivamente influenciada pela forma e o peso corporal.

O transtorno não ocorre exclusivamente durante episódios de Anorexia Nervosa.

\section{Especificação do Tipo:}

Tipo Purgativo: durante o episódio de Bulimia Nervosa, a pessoa se engaja regularmente em vômitos auto-induzidos ou uso de laxantes, diuréticos ou enemas.

Tipo Não-Purgativo: durante o episódio de Bulimia Nervosa, a pessoa tem usado outros comportamentos compensatórios inapropriados, tais como jejum ou exercício físico excessivo, mas não se engaja regularmente em vômitos auto-induzidos ou uso de laxantes, diuréticos ou enemas. 
como já citado, comportamentos sádicos com relação a pequenos animais entre seus irmãos.

Mãe tem relacionamento difícil com a paciente.

\section{Hábitos}

Tabagista de 40 cigarros/dia.

Nega abuso/dependência de outras substâncias lícitas ou ilícitas.

\section{Interrogatório Sobre os Diversos Aparelhos}

Refere queda de cabelo pronunciada.

\section{Exames Físico e Laboratoriais}

$\mathrm{PesO}=66 \mathrm{~kg}$

Altura $=1,58 \mathrm{~m}$

$\mathrm{IMC}=26,4 \mathrm{~kg} / \mathrm{m}^{2}$

Pressão Arterial $=100 \times 60 \mathrm{mmHg}$

Pulso $=88$ batimentos por minuto (Rítmicos)

Achados positivos: REG, descorada $++/++++$, desidratada $+/++++$

Abdômen: grande sobra cutânea pós-emagrecimento.

Membros Superiores: seqüela de cirurgia para correção de mal-formação secundária ao uso materno de talidomida.

\section{Exames Complementares:}

Hemácias $=3,72$ milhões $/ \mathrm{mm}^{3}$ (Valor de Referência $[\mathrm{VR}]=4,0$ a 5,4$)$

Hemoglobina $=11,4 \mathrm{~g} / \mathrm{dl}(\mathrm{VR}=12$ a 16$)$

Hematócrito $=34,1 \%(35$ a 47$)$

Leucócitos $=4,2 \mathrm{mil} / \mathrm{mm}^{3}(\mathrm{VR}=5$ a 10$)$;

Neutrófilos $=51,2 \%-2,2 \mathrm{mil} / \mathrm{mm}^{3}(\mathrm{VR}=2$ a 7$)$;

Linfócitos $=41,6 \%-1,8 \mathrm{mil} / \mathrm{mm}^{3}(\mathrm{VR}=0,5$ a 3,4$)$;

Monócitos $=5,3 \%-0,2 \mathrm{mil} / \mathrm{mm}^{3}(\mathrm{VR}=0,1$ a 1,0$)$;

Eosinófilos $=1,0 \%-0,0 \mathrm{mil} / \mathrm{mm}^{3}(\mathrm{VR}=0$ a 0,07$)$;

Basófilos $=0,8 \%-0,0 \mathrm{mil} / \mathrm{mm}^{3}(\mathrm{VR}=0$ a 0,2$)$

Eletroforese de Proteínas:

Proteínas $=100 \%-5,4 \mathrm{~g} / \mathrm{dl}(\mathrm{VR}=100 \%[6,0$ a 8,0$])$; Albumina $=52,7 \%-2,85 \mathrm{~g} / \mathrm{dl}(\mathrm{VR}=57$ a $63 \%[3,5 \mathrm{a}$ $5,0])$;

Alfa $\mathrm{l}=4,4 \%-0,24 \mathrm{~g} / \mathrm{dl}(\mathrm{VR}=3$ a $5 \%[0,2$ a 0,4$])$

Alfa $2=11,5 \%-0,62 \mathrm{~g} / \mathrm{dl}(\mathrm{VR}=6$ a $9 \%[0,4$ a 0,7$])$; Beta $=16 \%-0,86 \mathrm{~g} / \mathrm{dl}(\mathrm{VR}=10$ a $15 \%[0,6$ a 1,2$])$; $\mathrm{Gama}=15,5 \%-0,84 \mathrm{~g} / \mathrm{dl}(\mathrm{VR}=12$ a $21 \%[0,7$ a 1,6$])$

Proteínas (total e frações):

Proteínas Totais $=5,8 \mathrm{~g} / \mathrm{dl}(\mathrm{VR}=6,0$ a 8,0$)$;

Albumina $=3,3 \mathrm{~g} / \mathrm{dl}(\mathrm{VR}=3,5$ a 5,0$)$;

Globulinas $=2,5 \mathrm{~g} / \mathrm{dl}(\mathrm{VR}=2,5$ a 3,0$)$

Capacidade Total de ligação do Ferro e saturação do Ferro: diminuídas
Estradiol: diminuído;

Prolactina: elevada

\section{Exame Psíquico}

Sem alterações psicopatológicas significativas no momento da internação.

\section{Hipóteses Diagnósticas (DSM-IV) (20)}

(a) Transtorno Alimentar Não Especificado

(b) Transtorno de Personalidade Não Especificado

(c) Dependência de Nicotina

\section{Tratamento e Evolução}

O tratamento instaurado foi realizado por equipe multidisciplinar composta por psiquiatra (A.S.), nutricionista, psicóloga e equipe de enfermagem do AMBULIM-AMBESO no Instituto de Psiquiatria do HC-FMUSP.

Durante a internação, apresentou rituais associados a banhos e a limpeza desencadeados após a ingestão alimentar. A paciente referia sentir-se suja após comer, como se o alimento fosse algo imundo dentro de seu corpo. Devido a isso, foi adicionado o diagnóstico de Transtorno Obsessivo-Compulsivo (DSM-IV) (20).

Paciente recebeu alta 5 meses após a internação, com peso de $65 \mathrm{~kg}\left(\mathrm{IMC}=26,04 \mathrm{~kg} / \mathrm{m}^{2}\right)$, normalização dos parâmetros laboratoriais e de exame físico. À época, recebia fluoxetina $80 \mathrm{mg} / \mathrm{dia}$, nortriptilina $25 \mathrm{mg} /$ dia e periciazina $15 \mathrm{mg} /$ dia, sendo o segundo um antidepressivo. As fantasias violentas haviam remitido, assim como os banhos ritualizados. Mostrava-se colaborativa e motivada a continuar seu tratamento.

Durante o acompanhamento ambulatorial, a paciente passou a faltar às consultas com freqüência ou retornava esporadicamente, a não fazer uso da medicação, a restringir a alimentação e a provocar vômitos novamente. Não houve retorno de fantasias e atitudes sádicas. Este quadro provocou piora acentuada dos parâmetros laboratoriais e diminuição do peso.

A família foi convocada e a reversão parcial da operação passou a ser discutida, após esta possibilidade ter sido sugerida pela equipe cirúrgica, com o intuito de estabilizar o quadro clínico. Sendo assim, como tanto a paciente quanto a família não concordavam com a retirada do anel que limita a passagem alimentar, a paciente procurou outro profissional, externo ao Grupo.

Sete meses depois, a paciente voltou a procurar seu psiquiatra do AMBULIM-AMBESO, sem medicação. Mantinha o quadro alimentar descrito e a conseqüente inadequação de parâmetros de exames 
físico e laboratoriais, de gravidade maior do que aquela à época da internação, e apresentava recrudescência de fantasias sádicas. Foram introduzidos mirtazapina e olanzapina (na dose de $60 \mathrm{mg}$ e $10 \mathrm{mg}$, respectivamente, ao dia), o primeiro um antidepressivo e o segundo um neuroléptico atípico. Houve discreta e insuficiente melhora do quadro alimentar e remissão do quadro de fantasias.

Novamente, iniciou-se a discussão da reversão parcial da operação, porém, devido à investigação de tumores em mamas, esta discussão foi temporariamente adiada. A paciente foi internada por equipe profissional externa ao Grupo para sonoterapia (eufemismo habitualmente utilizado para a prática de eletroconvulsoterapia, porém os familiares negam ter sido este o procedimento) devido à ideação suicida desencadeada pela investigação ginecológica.

Até a redação final deste artigo, a paciente continuava internada em outro serviço.

\section{DISCUSSĀO}

A obesidade, enquanto doença crônica, tem sua busca de resultados, em qualquer modalidade terapêutica, centrada na mudança da morbi-mortalidade de longo prazo (15).

Procedimentos cirúrgicos passaram a ser introduzidos há várias décadas, porém apenas nos últimos 25 anos com resultados adequados, ou seja, redução substancial do peso e conseqüente diminuição da prevalência de doenças associadas à obesidade, tais como diabetes, apnéia obstrutiva do sono, hipoventilação, doenças ortopédicas ou reumáticas degenerativas, doença coronariana, miocardiopatia hipertrófica, colelitíase, além da melhoria da qualidade de vida em função da facilidade de locomoção corporal e da diminuição dos prejuízos psicossociais $(14,25)$.

Tabela 2. Critérios diagnósticos para Anorexia Nervosa (DSM-IV).

\section{Anorexia Nervosa:}

Recusa em manter o peso corporal dentro ou acima do normal para a idade e altura (abaixo de $85 \%$ do esperado).

Intenso medo de ganhar peso ou de tornar-se gordo, mesmo quando abaixo do peso normal para a idade e altura.

Distorção na maneira como percebe o próprio peso ou sua silhueta, não reconhecendo estar abaixo do peso normal.

Se mulheres após a menarca, amenorréia por pelo menos três ciclos menstruais
A indicação mais aceita é a presença de obesidade grau III (IMC> $40 \mathrm{~kg} / \mathrm{m}^{2}$ ). Quando, no entanto, o IMC está entre 35 e 40 na presença de co-morbidez de elevado risco de morbi-mortalidade, o procedimento cirúrgico também está indicado (14).

A mortalidade operatória está abaixo de $0,5 \%$ em centros especializados. Os efeitos colaterais mais comuns das técnicas de restrição gástrica são a intolerância a alimentos sólidos, vômitos, síndrome do empachamento (dumping syndrome), obstipação $\mathrm{e}$ queda de cabelo.

Complicações psiquiátricas são pouco conhecidas e, embora se estime que a incidência de depressão está em torno de $15 \%$, esses dados merecem melhor avaliação metodológica (16). Uma complicação em potencial que não pode ser esquecida é a possibilidade de suicídio $(27,28)$.

Estudando os benefícios psicológicos e mudança de estilo de vida resultantes da cirurgia de bypass gástrico, Waters e cols. (8) afirmam que esses são mais sentidos entre 6 e 12 meses de seguimento pós-cirúrgico, mas estacionam ou regridem ao final do segundo ano após a operação. No entanto, alguns anos depois, Powers e cols. (9) encontraram resultados satisfatórios em até 5,7 anos de seguimento.

Embora os relatos de Anorexia Nervosa e Bulimia Nervosa no seguimento pós operações bariátricas ainda sejam raros, o risco do desenvolvimento de um transtorno alimentar devia ser lembrado nessa situação. $\mathrm{O}$ transtorno alimentar aqui apresentado não preenche todos os critérios para Anorexia Nervosa ou Bulimia Nervosa (tabelas 1 e 2), podendo, no entanto, ser compatível com Transtorno Alimentar Sem Outra Especificação (tabela 3), exibindo um quadro com importantes alterações no comportamento alimentar $\mathrm{e}$ na preocupação excessiva com a perda de peso associadas a uma distorção da imagem corporal. O caso nos alerta para a necessidade de pesquisar sintomas mesmo sem a presença da síndrome completa.

Tabela 3. Critérios diagnósticos para Transtorno Alimentar Sem Outra Especificação (DSM-IV).

Transtorno Alimentar Sem Outra Especificaçāo (citamos apenas estes dois critérios que são os importantes para a discussão):

Para mulheres, todos os critérios para Anorexia Nervosa são encontrados, exceto que as menstruações são regulares.

Todos os critérios para Anorexia Nervosa são encontrados, exceto que, a despeito da significante perda de peso, o peso atual está dentro do normal.

Arq Bras Endocrinol Metab vol 48 n 4 Agosto 2004 
$\mathrm{Na}$ avaliação pré-cirúrgica, é importante pesquisar a presença de Transtorno da Compulsão Alimentar Periódica, o qual tem uma prevalência maior entre obesos mórbidos (10,29-31), e esse transtorno pode permanecer ativo, comprometendo o resultado pós-cirúrgico. Outra preocupação deve ser a imagem corporal, a qual sofre mudanças após a operação e pode estar distorcida nesses pacientes $(22,23)$. Essa mudança pode ser positiva, como a melhora na vida sexual, mas a presença de distorção da imagem corporal aumenta o risco de transtornos alimentares no pósoperatório.

A evolução do caso por nós apresentado mostra a dificuldade que existe para tratar pacientes com transtornos alimentares, mesmo num caso atípico como este e no qual o IMC está dentro de parâmetros adequados ou com discreto sobrepeso.

Cremos que o importante aqui é a discussão da psicopatologia essencial do quadro clínico. Os critérios diagnósticos são muito úteis em pesquisa e por isso tendem a ser rígidos. Se formos segui-los para o diagnóstico do caso em questão ou para muitos casos diagnosticados clinicamente como Anorexia Nervosa ou Bulimia Nervosa, poderíamos deixar de lado casos graves como este. A paciente já havia perdido mais de $50 \%$ de seu peso inicial e, no entanto, continuava desejando perder mais e utilizando recursos purgativos para obter esse resultado. Apenas esse comportamento já deve nos deixar atentos para os riscos de uma evolução para um transtorno alimentar.

Apenas durante a internação, obteve-se a aderência da paciente ao tratamento, que, no entanto, desapareceu rapidamente no seguimento ambulatorial, semelhante ao que ocorreu no segundo caso relatado por Atchison e cols. (5).

Nos antecedentes individuais da paciente, há o diagnóstico de Bulimia Nervosa e isso deve nos deixar alertas para o desenvolvimento de um transtorno alimentar após a operação. $\mathrm{O}$ uso de medicações antiobesidade e de dietas variadas é comum à grande maioria dos obesos mórbidos (14), mas são comportamentos que se somam na preocupação com o resultado pós-cirúrgico. Tais condições não deveriam excluir pacientes obesos da possibilidade do tratamento cirúrgico. É importante salientar que a presença de um quadro psiquiátrico no pré-operatório num paciente com obesidade grau III não deve ser encarado como contra-indicação para uma operação que pode ser a diferença entre oferecer uma melhor qualidade de vida ou esperar o surgimento de uma complicação potencialmente letal, graças às patologias comumente associadas. A contra-indicação poderá ser relativa, depen- dendo do quanto o transtorno psiquiátrico interfira na compreensão do tratamento (14). Na literatura pertinente às operações bariátricas, critérios de exclusão psiquiátrica foram gradativamente abandonados ao longo dos últimos dez anos $(14,17,18,21,26)$.

Black e cols. (19), estudando 88 pacientes que procuraram tratamento cirúrgico para obesidade, encontraram uma maior probabilidade de dependência de fumo e de transtornos de personalidade de vários tipos. O caso por nós relatado também apresenta um transtorno de personalidade. Vale ressaltar que esse tipo de diagnóstico requer a presença de certas características ou comportamentos que são estranhos aos da cultura onde a pessoa está inserida, e ocorrem desde a adolescência ou início da vida adulta de uma forma pervasiva e independente de fatores internos ou externos, como mostram os critérios do DSM-IV (20) na tabela 4. No entanto, mesmo entre os psiquiatras, esse é um diagnóstico muito discutido quanto à sua validade, pois especula-se que muitos desses transtornos de personalidade poderiam ser a manifestação diferenciada de algum transtorno psiquiátrico de origem orgânica. No caso de nossa paciente, o transtorno de personalidade não é passível de especificação pelo DSM-IV (20).

Reversão de operações bariátricas têm sido pouco freqüentes ou têm sido realizadas por distúrbios inerentes à própria técnica cirúrgica, tanto que não encontramos dados na literatura a respeito, e os relatos são anedóticos. No caso da nossa paciente, a reversão passou a ser discutida para melhorar a alimentação e a condição clínica, mas acabou não se consumando.

O trabalho pré e pós-cirúrgico deve incluir uma adequada atuação de uma equipe multiprofissional com psiquiatra conduzindo a avaliação diagnóstica e o tratamento farmacológico, psicólogos administrando psicoterapia individual, conjugal ou familiar, com orientações específicas sobre a operação, nutricionista e endocrinologista, tudo visando discutir e adequar as expectativas do paciente às limitações do tratamento cirúrgico muitas vezes concebido por ele como milagroso, além de oferecer alternativas de prazer diferentes do ato de comer. Há que se prevenir o abandono do seguimento pós-cirúrgico com o paciente acreditando já ter resolvido todos os seus problemas com a cirurgia. As mudanças ocorridas após a operação são muitas, tanto em nível comportamental como em nível físico e psíquico, o que pode trazer surpresas para a equipe multidisciplinar, como ocorreu no caso em questão. Tais surpresas podem ser evitadas freando-se a ansiedade em resolver a obesidade através da operação e fazendo-se uma anamnese préoperatória mais completa, cercando-se toda e qualquer possibilidade de alterações ligadas à alimentação. 
Tabela 4. Critérios diagnósticos gerais para um Transtorno de Personalidade (DSM-IV).

Um padrão contínuo de experiência e comportamento próprios que diverge marcadamente do esperado para a cultura do indivíduo. Este padrão se manifesta em duas (ou mais) das seguintes áreas:

- Cognição (isto é, a maneira de perceber e interpretar a si próprio, aos outros e aos eventos);

- Afetividade (isto é, a variação, a intensidade, a labilidade e a apropriação da resposta emocional);

- Funcionamento interpessoal;

- Controle do impulso.

O padrão contínuo é inflexível e pervasivo em uma larga variação de situações pessoais e sociais.

O padrão contínuo leva a uma angústia clinicamente significativa ou prejuízo nas áreas social e ocupacional, ou em outras importantes áreas do funcionamento.

O padrão é estável e de longa duração e seu início pode ser traçado no passado, pelo menos na adolescência ou no início da vida adulta.

O padrão contínuo não é melhor categorizado como uma manifestação ou conseqüência de um outro transtorno mental.

O padrão contínuo não é devido aos efeitos fisiológicos diretos de uma substância (por exemplo, uma droga de abuso ou uma medicação) ou a uma condição médica geral (por exemplo, trauma craniano).

A partir de uma anamnese pré-operatória, a presença de um transtorno alimentar deve forçosamente levar a um seguimento psiquiátrico pós-operatório para a detecção precoce de um agravamento do mesmo, pois a prevalência de tais transtornos é maior em pacientes com obesidade grau III e, na presença deles, o risco de complicações é cerca de quatro vezes maior (8-13). No entanto, não há necessidade de acompanhamento psiquiátrico de todos os obesos operados. Certos casos podem ser indicados para o tratamento cirúrgico pelos próprios diagnósticos psiquiátricos que apresentam, como depressão por exemplo, se bem que raramente a indicação será apenas por algum diagnóstico psiquiátrico associado a um IMC elevado.

\section{REFERÊNCIAS}

1. Bonne O, Bashi R, Berry E. Anorexia Nervosa following gastroplasty in the male: two cases. Int J Eat Disord 1996; 19:105-8.

2. Fichter MM, Daser C. Symptomatology, psychosexual development and gender identity in 42 anorexic males. Psychol Med 1987;17:409-18.

3. Herzog DB, Norman DK, Gordon C, Pepose M. Sexual conflict and eating disorders in 27 males. Am J Psychiatry 1984;141:989-90.

4. Carlat DJ, Camargo CA Jr, Herzog DB. Eating disorders in males: a report on 135 patients. Am J Psychiatry 1997; 154:1127-32.

5. Atchison M, Wade T, Higgins B, Slavotinek T. Anorexia Nervosa following gastric reduction surgery for morbid obesity. Int J Eat Disord 1998;23:111-6.
6. Scioscia TN, Bulik CM, Levenson J, Kirby DF. Anorexia Nervosa in a 38 -year-old woman 2 years after gastric bypass surgery. Psychosomatics 1999;40:86-8.

7. Algazi LP. Transactions in a support group meeting: a case study. Obes Surg 2000;10:186-91.

8. Waters $G$, Pories $W$, Swanson $M$, et al. Long-term studies of mental health after the Greenville gastric bypass operation for morbid obesity. Am J Surg 1991;161:1547.

9. Powers PS, Rosemurgy A, Boyd F, Perez A. Outcome of gastric restriction procedures: weight, psychiatric diagnoses, and satisfaction. Obes Surg 1997;7:471-7.

10. Powers PS, Perez A, Boyd F, Rosemurgy A. Eating Pathology before and after bariatric surgery: a prospective study. Int J Eat Disord 1999;25:293-300.

11. Kalarchian M, Wilson $G$, Brolin R, et al. Binge Eating in bariatric surgery patients. Int J Eat Disord 1998;23:8992.

12. Hsu L, Betancourt S, Sullivan S. Eating disturbances before and after vertical banded gastroplasty: a pilot study. Int J Eat Disord 1996;19:23-34.

13. Hsu LK, Sullivan SP, Benotti PN. Eating Disturbances and outcome of gastric bypass surgery: a pilot study. Int J Eat Disord 1997;21:385-90.

14. ASBS - American Society for Bariatric Surgery. Rationale for the surgical treatment of morbid obesity. http://www.asbs.org/html/ration.html Updated 04/06/98, Reviewed 04/08/98.

15. Lyznicki JM, Young DC, Riggs JA, Davis RM; Council on Scientific Affairs, American Medical Association. Obesity: assessment and management in primary care. Am Farm Physician 2001;63:2185-96.

16. Kral JG. Surgical treatment of obesity. In: Kopelman PG, Stock MJ, eds. Clinical obesity. Oxford: Blackwell Science, 1998. p.545-63.

Arq Bras Endocrinol Metab vol 48 n 4 Agosto 2004 
17. NIH Consensus Statements. Gastrointestinal surgery for severe obesity, 1991.

18. Patient selection for bariatric surgery, International Federation of Surgery for Obesity (IFSO) http://www.obesity-online.com/ifso

19. Black DW, Goldstein RB, Mason EE. Prevalence of mental disorder in 88 morbidly obese bariatric clinic patients. Am J Psychiatry 1992; 149:227-34.

20. Diagnostic and statistical manual of mental disorders: DSM-IV - 4th ed. American Psychiatric Association, 1994

21. Segal A, Azevedo AP, Libanori HT. Bariatric surgery in a patient with possible psychiatric contraindications. Obes Surg 2002; 12:598-601.

22. Camps MA, Zervos E, Goode S, Rosemary AS. Impact of bariatric surgery on body image perception and sexuality in morbidly obese patients and their partners. Obes Surg 1996;6:356-60.

23. Adami GF, Meneghelli A, Bressani A, Scopinaro N. Body image in obese patients before and after weight reduction following bariatric surgery. J Psychosom Res 1999;:46:275-81.

24. Maddi SR, Fox SR, Khoshaba DM, Harvey RH, Lu JL, Persico M. Reduction in psychopathology following bariatric surgery for morbid obesity. Obes Surg 2001;11:680-5.

25. Balsiger BM, Murr MM, Poggio JL, Sarr MG. Bariatric Surgery for weight control in patients with morbid obesity. Med Clin North Am 2000;84:477-89.
26. Coutinho W. Consenso Latino-Americano de Obesidade. Arq Bras Endocrinol Metab 1999;43:21-67.

27. Higa KD, Boone KB, Ho T. Complications of the laparoscopic Roux-en-Y gastric bypass: 1,040 patients - what have we learned? Obes Surg 2000;10:509-13.

28. Hsu LK, Benotti PN, Dwyer J, Roberts SB, Saltzman E, Shikora $S$, et al. Nonsurgical factors that influence the outcome of bariatric surgery: a review. Psychosom Med 1998;60:338-46.

29. Fontenelle LF, Mendlowicz VM, de Menezes GB, Papelbaum M, Freitas SR, Godoy-Matos A, et al. Psychiatric comorbidity in a Brazilian sample of patients with bingeeating disorder. Psychiatry Res 2003; 119:189-94.

30. Borges MBF. Estudo do Transtorno da Compulsão Alimentar Periódica em população de obesos e sua associação com depressão e alexitimia (Disser tação). São Paulo: Universidade Federal de São Paulo: 1998.

31. Borges MB, Jorge MR, Morgan CM, da Silveira DX, Custodio $\mathrm{O}$. Binge-eating disorder in Brazilian women on a weight-loss program. Obes Res 2002;10:1127-34.

Endereço para correspondência:

Táki Athanássios Cordás

Rua Capote Valente 432, conj. 35

05409-001 São Paulo, SP

e-mail: cordas@usp.br 